$\xi=-1$ 圆

\title{
Development of an experimental single-link flexible manipulator system
}

\author{
Hanim Mohd Yatim ${ }^{*}$, Intan Zaurah Mat Darus ${ }^{2}$ \\ ${ }^{1}$ Universiti Kuala Lumpur, Malaysian Institute of Industrial Technology, Bandar Seri Alam, Johor, Malaysia \\ ${ }^{2}$ Faculty of Mechanical Engineering, Universiti Teknologi Malaysia, Skudai, Johor, Malaysia \\ *Corresponding author E-mail: hanim@unikl.edu.my
}

\begin{abstract}
Demand for the applications of flexible manipulator due to their benefits has received attention from the industries. However, the flexibility of flexible manipulator has resulted in structural vibration and needs to be studied accordingly. This paper presents the development of a laboratory facility constituting of single-link flexible manipulator system. A new experimental rig flexible manipulator system constrained to move horizontally was designed, developed and fabricated. The experimental equipment setup and method of capturing data are presented. Experimental works have been done to highlight the effect of forces on hub-angle and end-point vibration of the flexible manipulator. Impact test was carried out to identify the dominant mode of vibration of the flexible manipulator. Result from the impact test was compared with the experimental data recorded for model validation and verification. The experimental result demonstrated that the reasonable accuracy with only $1.31 \%$ of error was obtained for the dominant mode of vibration. The experimental rig developed incorporating the sensors and actuators deployed may direct future researchers towards significant applications of flexible manipulator in the industrial sector and to promote better productivity.
\end{abstract}

Keywords: Flexible Manipulator; Single-link Manipulator, Laboratory design

\section{Introduction}

The manipulator is a mechanical part, comprising of many segments, intended for a widely range applications. It is designed to increase the productivity and suitable in different tasks and environment. Examples can be found such as robot arms, satellite, mobile manipulator, flexible hand rest, material handling systems, automated manufacturing systems, and fundamentals of automated guided vehicle systems.

Traditionally, rigid manipulators are designed and built in a manner that maximize stiffness to minimize vibration and allow stable performance. High stiffness is achieved by using heavy material that limits the rapid motion of the manipulator with shorter reach, required expensive construction and consumes more energy during application (Tokhi et.al., 2001; Vakil; 2008). Moreover, there are many situations that prevent the use of industrial rigid manipulator configurations especially when the workspace is constrained and assembly processes are required. Therefore, flexible manipulator systems have received substantial attention to reduce the weight of mechanical structures and thus improve the manipulator performance. It offers several advantages over their rigid counterparts including lighter weight, lower energy consumption, faster system response, safer operation due to reduced inertia, smaller actuator requirement, low-strength mounting and low-rigidity requirement, less bulky design and are more transportable and maneuverable (Mohamed et al., 1996; Choi et al.1999; Tokhi et al., 2001).

However, flexible manipulators are known to demonstrate an oscillatory behavior when subjected to disturbance forces due to manipulator flexibility (Abdul Razak, 2007). If the advantages of flexible manipulator are not to be sacrificed, thus the issue of flex- ible manipulator design can be primarily cater by the design of controllers to compensate for the structural flexibility (Tokhi and Azad, 1996). Stringent requirements on precise positioning and suppression of vibration have motivated many researchers in an attempt to propose efficient control strategies for flexible manipulator system.

It has been shown that feedforward control is adequate for satisfactory performance as reported in several studies (Rudrusamy, 2005; Alam and Tokhi, 2008). However, feedforward control methods limits the parametric changes and disturbances (Phan et al. 2009). With regard to feedback control, a number of different control strategies were introduced to overcome this drawback (Rovner and Franklin, 1988; Ryu et al. 2000; Ho and Tu, 2006; Ahmad and Mohamed, 2008; Maouche and Attari, 2008). In addition to measuring joint positioning and vibration suppression, it is desirable to employ the smart material in line with sensing and control that are done locally by smart materials for different physical properties. While retaining the advantages of lightweight flexible structures, it has additional sensing and control capabilities.

This argument for control purposes leads to requirement of a suitable measuring system at the free-end of the flexible manipulator. Choice of suitable actuator and sensing equipment for the control mechanisms are need to be properly select.

This paper presents the design of a laboratory facility for experimental study of a horizontally constrained single-link flexible manipulator. The concept of mechatronics comprises the integration of mechanical, instrumentation, computer and control engineering. The principal of the design presented are the decentralizing control schemes that leads to employment of piezoelectric (PZT) smart material as vibration controller at the flexible link manipulator. 


\section{Mechatronic System}

The flexible manipulator used in this study is an aluminum link which is pinned at the hub and free at the other end. The mechatronic system considered consists of integration of the mechanical, instrumentation and computer aspects. Firstly, the mechanical components of the flexible manipulator rig were developed and fabricated. Then, the instrumentation components includes sensor and actuators were chosen according to the control schemes of flexible single-link manipulator system. Next, the experimental procedure and system integration used to conduct the experiment was discussed.

\subsection{Mechanical System}

A single link thin aluminum alloy of flexible manipulator is considered. The dimension of flexible manipulator used is $600 \mathrm{~mm}$ in length, $40 \mathrm{~mm}$ in width and $1.5 \mathrm{~mm}$ in thickness. It is manufactured using an aluminum plate of $2710 \mathrm{~kg} / \mathrm{m}^{2}$ density and $71 \mathrm{GPa}$ Young Modulus. An orthographic view of the finalized design is shown in Figure 1. The flexible manipulator is pinned and attached to the motor at one end and free at the other end. The manipulator model was designed to move in a horizontal plane. A 3D representation and the exploded view of the experimental rig structure construction are shown in Figures 2 and 3 respectively.

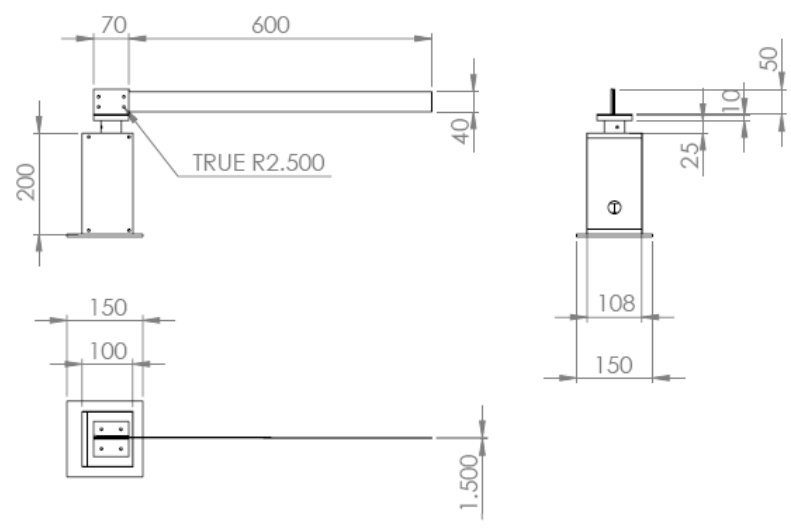

Fig. 1: An orthographic drawing of the flexible manipulator

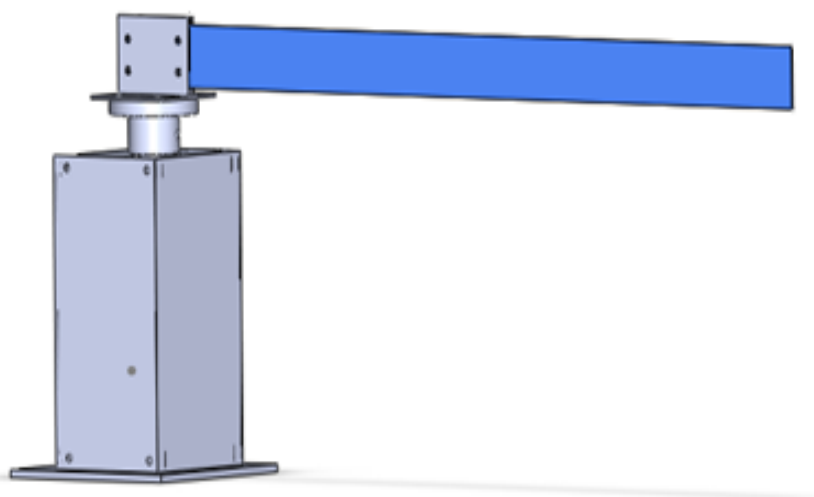

Fig. 2: Isometric model of the experimental rig

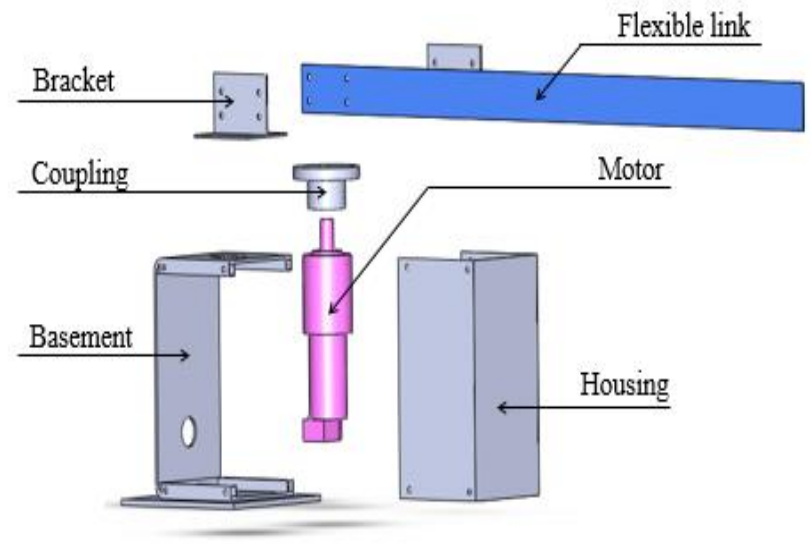

Fig. 3: Exploded view of the experimental rig

\subsection{Instrumentation System}

The comprehensive instrumentation component considered in this study includes sensors, actuators and a computer with programmable software. In this study, accelerometer and encoder is used as sensors while piezoelectric and DC motor acts as actuators. Data acquisition system (DAQ) from National Instrument (NI) is selected for interface between measurement hardware and programmable software.

The actuation system that was used to move the link consists of a 150 Watt DC motor manufactured by Maxon motor company of type RE 40 as shown in Figure 4. The motor was attached at the hub serves as an actuator to drive the flexible manipulator. This motor has a compact shape with mass of $480 \mathrm{~g}$, can be easily engaged with the mechanical design of manipulator and compatible with the load of structure. In addition, it was paired together with a planetary gearhead of 21:1 reduction ratio by Maxon motor Company of type GP 52C of $\emptyset 52 \mathrm{~mm}$ in order to increase the efficiency of the motor. Gearing system is engaged to the motor in order to amplify the output torque to the load and thus minimize the input power.

In this research, a motor driver model EPOS2 50/5 as recommended by Maxon Motor was employed and it was designed to control the Maxon DC motor as shown in Figure 5. The motor driver is responsible to regulate the current that will be passed to the DC motor in order to control the speed and torque of the motor. It is small with powerful 4-quadrant PWM servo controller utilized for the highly proficient control of DC motors that can easily integrate into the PC and promote easy programming with available software. The operating mode can be control using the EPOS Studio software package. This tool used to configure the controller including configuration of digital and analog inputs and outputs. In order to acquire the angular position input signal from motor, an encoder was used as a sensor as shown in Figure 6. Encoder model HEDL 5540 from Maxon is chosen for closed-loop control purposes. The function of encoder is to capture the position of the motor or to be specific the angle of the hub with precision of 500 counts per turn. With a 21.1 gear reduction will result in a resolution of 23.81 counts per degree of revolution of output shaft. The encoder sends a digital signal which directly connected through Maxon EPOS3 50/5 controller.

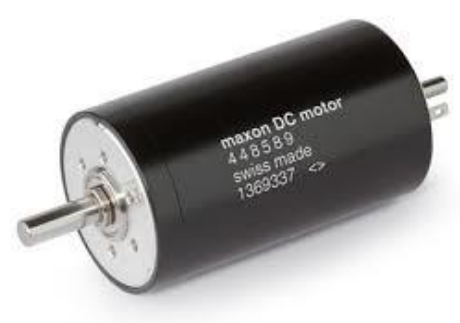

Fig. 4: Maxon DC motor of RE 40 


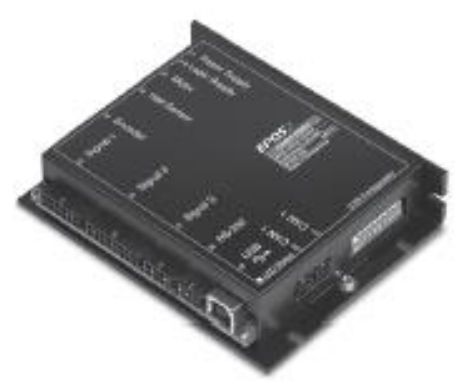

Fig. 5: Maxon EPOS2 50/5

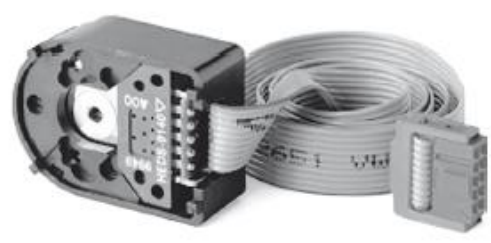

Fig. 6: Maxon Encoder HEDL 5540

In this study, single axis ICP accelerometer with $100 \mathrm{mV} / \mathrm{g}$ sensitivity and $50 \mathrm{~g}$ measuring range as shown in Figure 7 is chosen to serves as a sensor in order to acquire the vibration input. Accelerometer works when there is subjected to acceleration or vibration, an electrical charge will be generated. This electrical charge will be converted through signal conditioning unit into signal that can be detected by a data acquisition system which is voltage. This device was easily connected to the PC via SCC-ACC01 signal conditioning unit and connector block of NI-SCC68. Since the maximum vibration of flexible manipulator was occur at endpoint, the accelerometer was placed at this point as illustrated in Figure 8. A very light weight sensor weighing only $4 \mathrm{~g}$, it's suitable for compact design application with weight restriction and will not influence the flexible link dynamics.

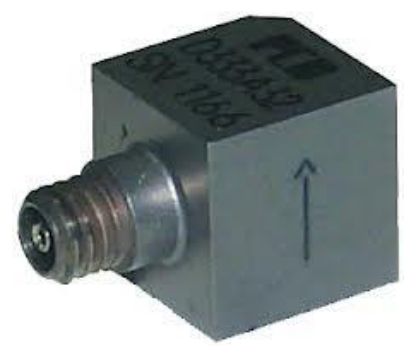

Fig. 7: Single axis ICP accelerome ter

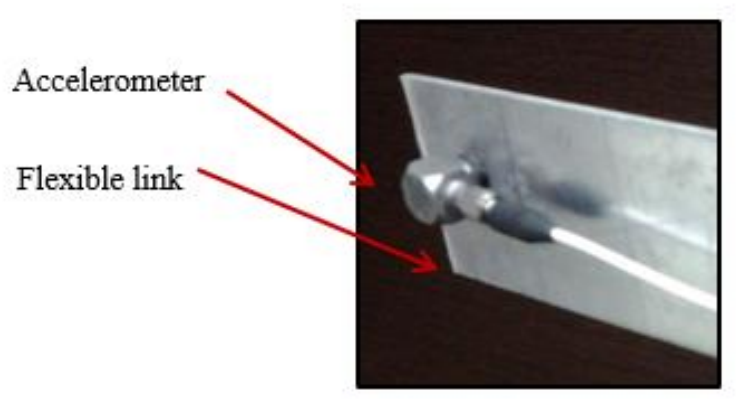

Fig. 8: Accelerometer bonded at endpoint of flexible manipulator

P-876.A15 DuraAct piezoelectric actuator as shown in Figure 9 was employed in this study as an actuator in order to produce the control signal. This piezoelectric patch transducer can generate mechanical deformation when voltage is applied and vice versa and capable in generating voltage when subjected to applied stress.
It was made of piezoelectric materials called as lead zirconatetitanate (PZT). It offers several advantages includes rugged and compact design, high flexibility, fast response, can be affixed to the structures, and excellent actuation capabilities. The technical specifications of the piezo actuator used are listed in Table 1 (Physik Instrumente, 2012). Figure 10 shows the PZT actuators mounted on the manipulator. PZT actuator is required to perfectly mount to the link surface, so that the actuator loading can be evenly distributed over its relatively wide dimension.

Table 1: Specification of the piezoelectric actuator

\begin{tabular}{|l|l|}
\hline Parameter & Specification \\
\hline Dimensions $(\mathrm{mm})$ & $61 \times 35 \times 0.8$ \\
\hline Mass $(\mathrm{g})$ & 7.2 \\
\hline Bending radius $(\mathrm{mm})$ & 70 \\
\hline Supply voltage $(\mathrm{V})$ & -250 to 1000 \\
\hline Blocking force $(\mathrm{N})$ & 775 \\
\hline
\end{tabular}

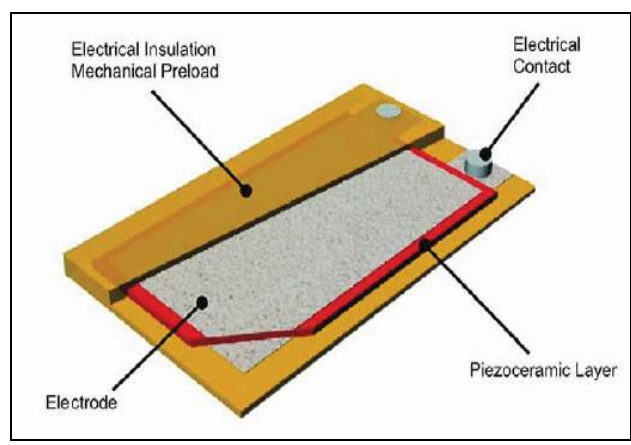

Fig. 9: P-876.A15 DuraAct piezoelectric patch

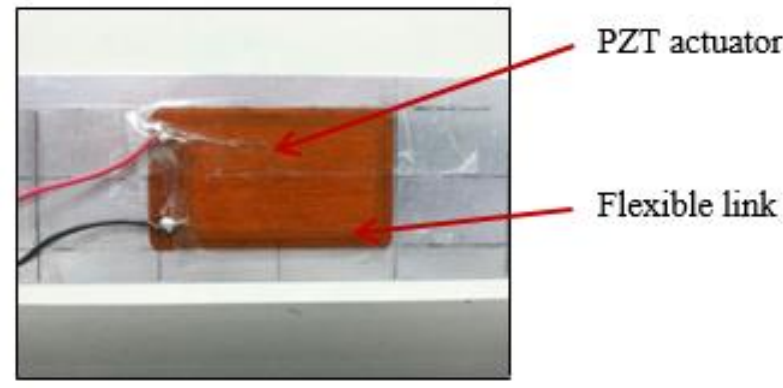

Fig. 10: $\mathrm{PZT}$ actuator of P-876.A15 mounted on the manipulator

The piezo amplifier of OEM E-835 with 10 voltage gain as shown in Figure 11 is employed to be paired with the DuraAct PZT actuator as it is designed purposely for this actuator. Function of piezo actuator amplifier is to amplify the voltage level from data acquisition system before passed it to the PZT actuator. The analog output voltage from SCC-68 of data acquisition system can create a voltage range between -10 to $+10 \mathrm{~V}$. Though, a voltage range of piezo actuator is within -250 to $+1000 \mathrm{~V}$. Therefore the piezo amplifier will amplify the voltage signal so that PZT actuator will works at its optimum performance.

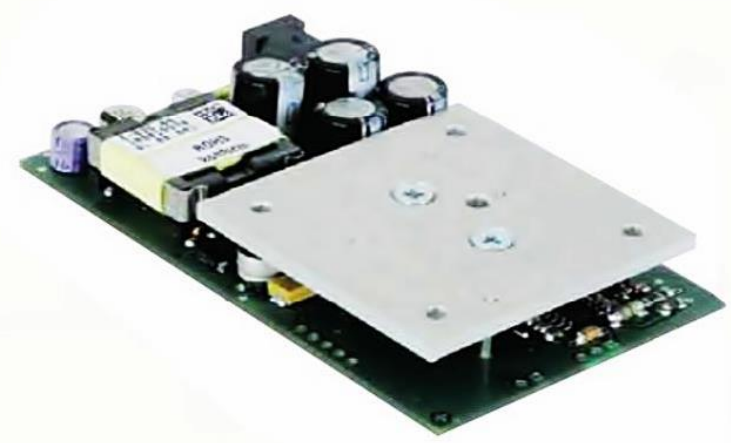

Fig. 11: Piezoelectric actuator amplifier type E-835 OEM 
Data acquisition system from National Instruments (NI) was used as the interface unit in this study. Its include data acquisition card model PCI-6259 and input output connector block SCC-68 series. This high end interface card was chosen due to its availability and compatibility. It can be easily integrate into the PC and can configure the input output from many sensors and actuators which suitable to be used for a wide applications. The SCC-68 has 32 analog input-output channels for easy input-output connections. It also has 4 expansion slots for analog input and digital input-output using signal conditioning modules which suitable for accelerometer modules. The PCI-6259 data acquisition card mounted inside the computer and connected to the SCC- 68 block. Maximum voltage range for analog input-output is from -10 to $10 \mathrm{~V}$ which meets the demand of this study for interfacing with PZT actuator and piezo amplifier. The data acquisition card of PCI-6259 and connector block SCC-68 are depicted in Figures 12 and 13 respectively.

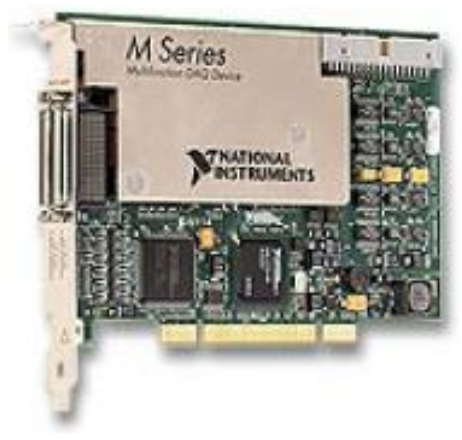

Fig. 12: NI PCI-6259

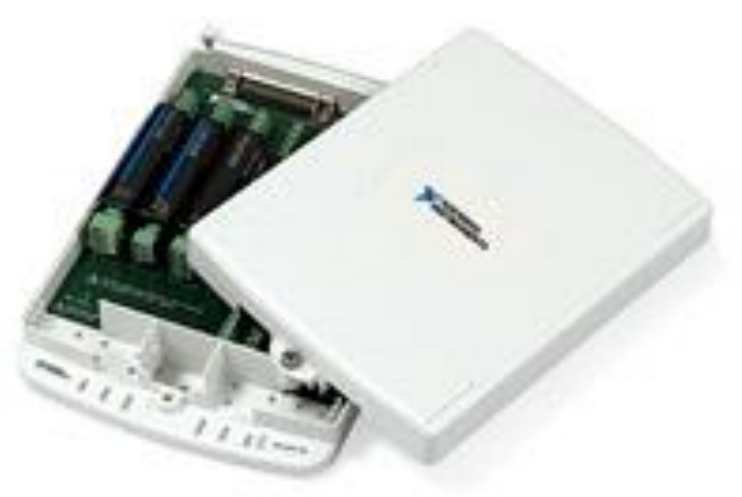

Fig. 13: Connector block SCC-68 series

\subsection{Software and Computer Control}

In this study, personal computer (PC) is used as a processer of the system. The purpose of PC is to send and receive data from the hardware. The specifications of the computer are Intel core I5, $2.93 \mathrm{GHz}$. MATLAB/Simulink is used as the environment for the development of the controller. The signal from the hardware is transmitted into MATLAB environment and used for the development of the controller. The version of the MATLAB is Version R2011a.

\section{System Integration}

Experimental study based on the schematic diagram shown in Figure 14 was conducted in order to acquire the input-output data and validate the effectiveness of the proposed controller.

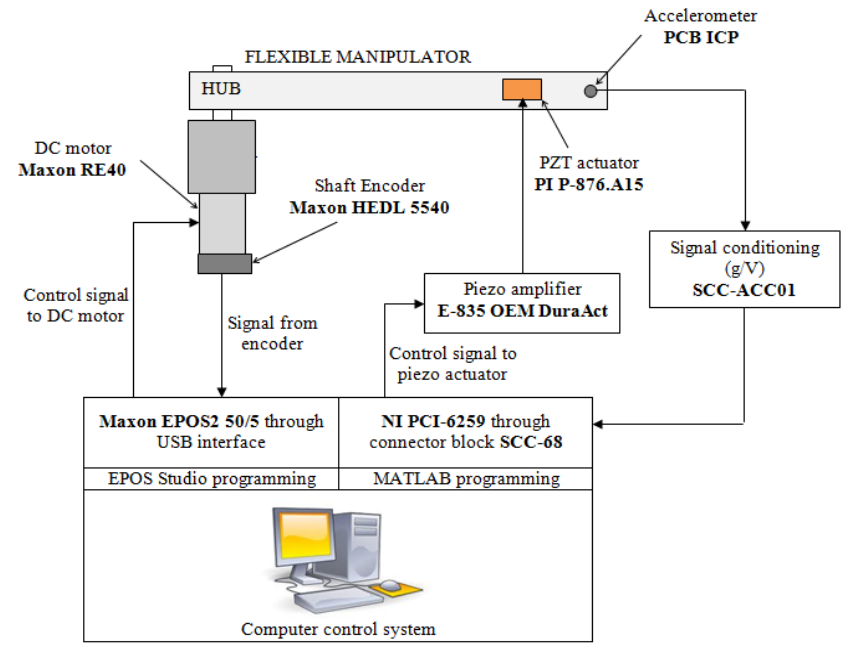

Fig. 14: Schematic diagram of the experimental set up

The aluminum link of $600 \times 40 \times 1.5 \mathrm{~mm}$ dimension was pinned at one end and free at other end. The DC motor was attached directly at the rotational axis to driven the flexible manipulator. Therefore, the speed and angle of motor displaced could be easily controlled via PC connection. The angle was sensed by the encoder that has 500 counts per turn which secured directly to the motor. The digital signal was then fed to the motor driver of EPOS2 50/5 that was interfaced to the PC via USB. EPOS2 50/5 acts as a data acquisition system that will measure and process the data signal from encoder and to the motor. EPOS Studio software provided by Maxon Motor was used as a platform in control programming of hub angle analysis. The integration of the DC motor, gearhead and encoder which connected to the PC through EPOS2 motor driver is shown in Figure 15.

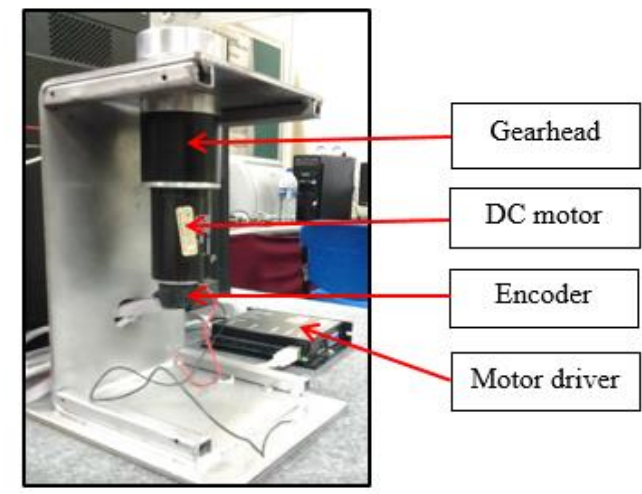

Fig. 15: Maxon DC motor, gearhead, and encoder attached together at hub and connected to the motor driver

Due to the flexibility of the manipulator, an unwanted vibration will be present and maximum at endpoint when the link was moved by the motor. This endpoint vibration will be measured by the accelerometer. The acceleration signal needs to pass through the signal conditioner SCC-ACC01 to produce appropriate voltage signal. Then, this signal is acquired by the NI DAQ and will be analyzed via PC using MATLAB environment. Later on, the control output signal was sent to the piezo actuator that was mounted on the surface of the flexible manipulator. Since output from NI DAQ is in the range between -10 to $+10 \mathrm{~V}$, a piezo amplifier was employed that has a gain of up to 10 and is capable to amplify the output voltage from -100 to $+100 \mathrm{~V}$, enough to actuate the piezo patch. The control algorithm is developed within MATLAB programming software where interfacing between the input-output can be made easily for endpoint vibration analysis. The experimental setup to measure and control the hub angle and endpoint vibration of a flexible manipulator is shown in Figure 16. 
The placement and interface between all sensors and actuators promote the decentralizing control schemes which sensing and control are done locally for different physical properties. Motor mounted at the hub with encoder sensor were measuring joint positioning while smart material PZT bonded to the surface of flexible link at endpoint with accelerometer sensor were utilized for vibration analysis purposes. Thus the complex system of flexible manipulator was treated as two single-input single-output (SISO) systems with two different control closed loops.

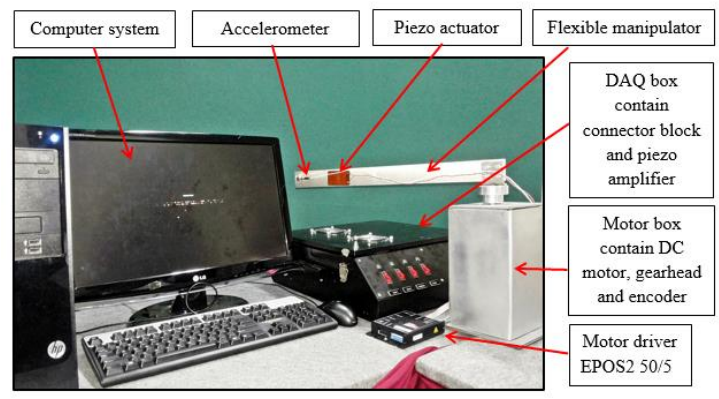

Fig. 16: Complete experimental rig

\section{Results and Findings}

In this section, results from experimental tests of the flexible manipulator are presented. System behavior at the hub and endpoint was observed and recorded. Validation of the developed experimental model was carried out by comparing the first three modes of vibration of the experimental output with the impact test result.

\subsection{Experimental Test}

A thin aluminum flexible manipulator was driven by a motor fed with a bang-bang torque input applied at the hub as shown in Figure 17. This type of input is applied to allow the link accelerate, decelerate and stop accordingly at the desired position. The response of the flexible manipulator is monitored for a duration of 4.5 second with a sampling time of $0.52 \mathrm{~ms}$.

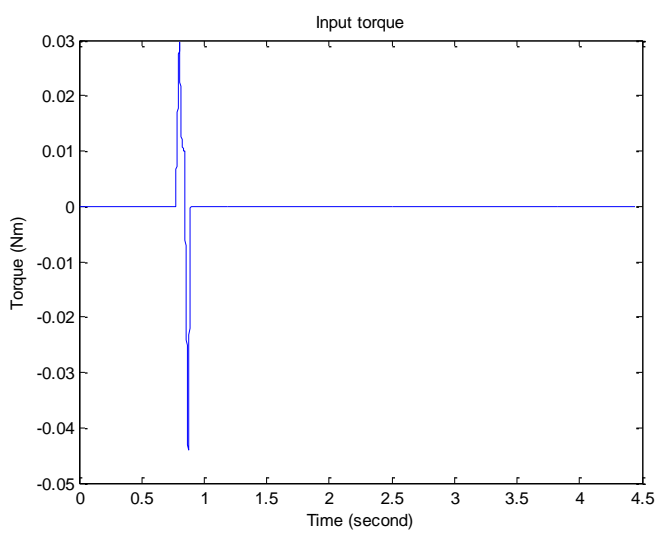

Fig. 17: Input torque bang-bang

An experimental test was conducted by moving the flexible manipulator rig to the desired angle of 8.5 degree. For this small angle displacement, an input torque of $\pm 0.03 \mathrm{Nm}$ was given. The open loop responses of the hub angle and acceleration at the free end of the flexible manipulator were recorded as depicted in Figures 18 and 19 respectively. For hub angle, the observation was completed by the encoder that was attached to the motor and the data was analyzed using EPOS Studio programming. Meanwhile, the accelerometer placed at the endpoint was responsible to record the endpoint acceleration and was analyzed in MATLAB environment.

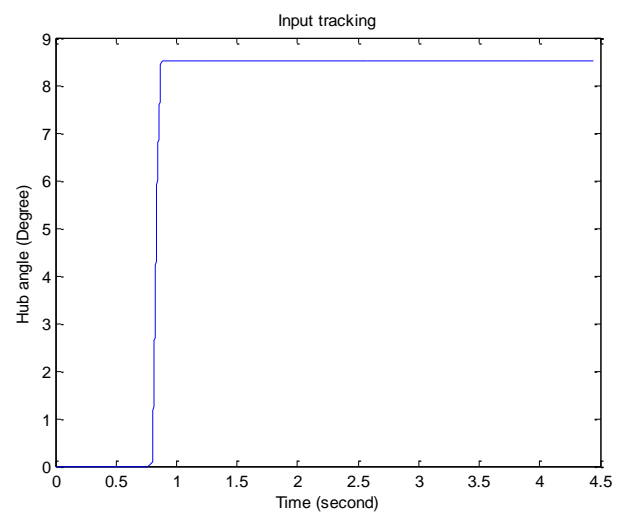

Fig. 18: Hub angle

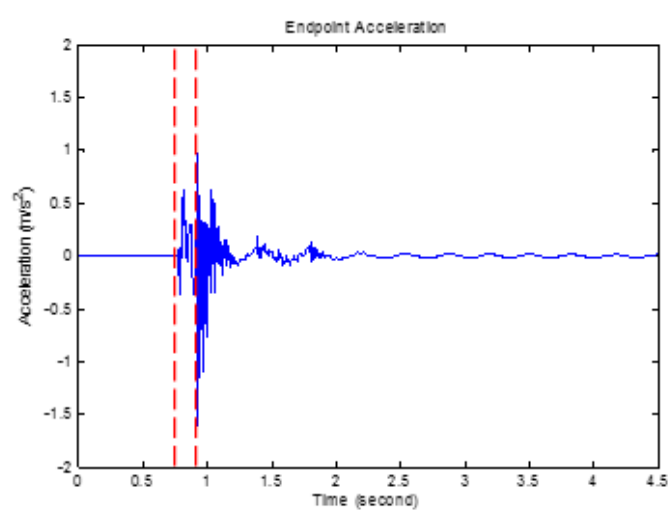

(a) Time domain

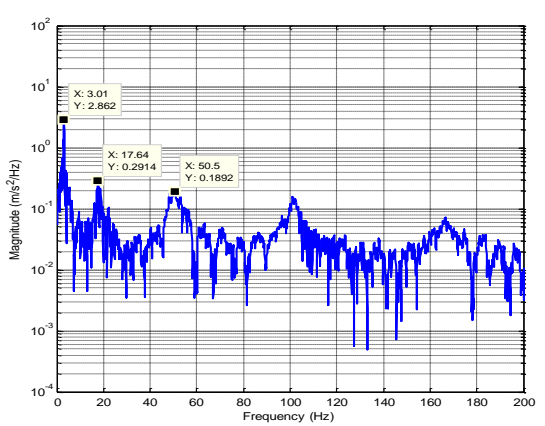

(b) Frequency domain

Fig. 19: Endpoint acceleration in (a) time and (b) frequency domain

Response of the hub angle with the given input in time domain is shown in Figure 18. The actual output of hub angle has remained steady at the desired position without overshoot. Figure 19 depicts the endpoint acceleration of flexible manipulator in time and frequency domain.

From Figure 19(a), two distinct high peaks can be distinguished as shown by the dotted lines. The first peak represents the moment flexible link started to move and second peak is during the deceleration of the link which finally stopped at desired position. The system behavior shows that it is consistently oscillated after stopping because there is no inclusion of damping effect or an active control. From Figure 19(b), the first three resonant modes recorded were at $3.01,17.64$ and $50.50 \mathrm{~Hz}$.

\subsection{Impact Test}

An impact test was carried out on the flexible manipulator where an impact was given on the bare structure by a hammer or load that swing freely from a certain height. The dynamic response was observed through the accelerometer during the impact test and data was analyzed using MATLAB software. The response from the free vibration test was recorded in time domain as shown in Figure 20. From Figure 21, the first three resonance frequencies for flexible manipulator were found to be at 3.05 , 18.31 and $53.10 \mathrm{~Hz}$, with the first mode as the dominant mode. 


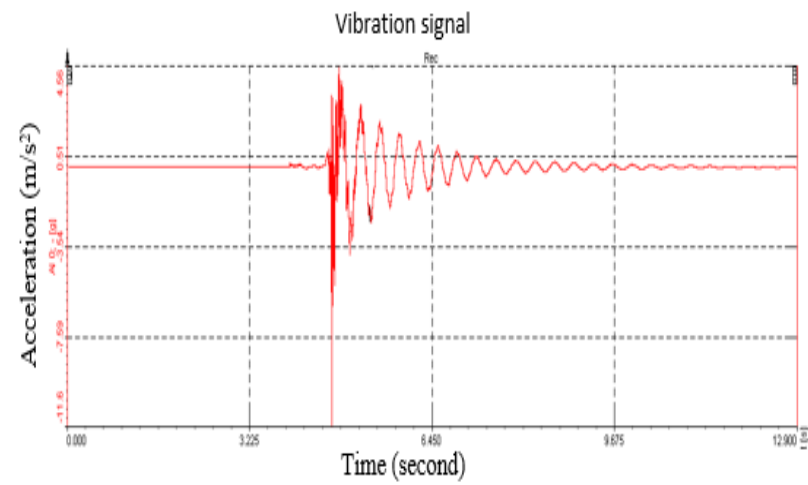

Fig. 20: Impact test in time domain

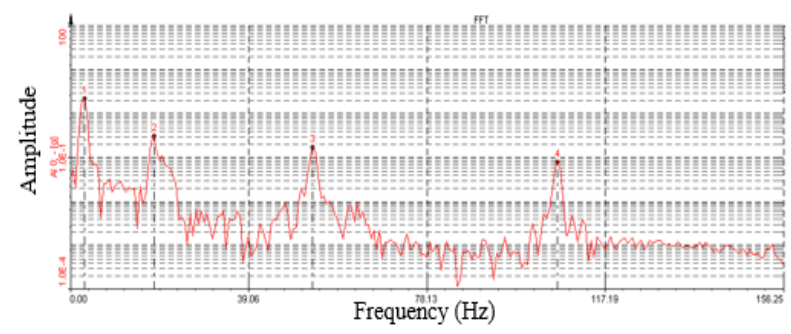

Fig. 21: Impact test in frequency domain

\subsection{Model Verification}

As in previous section, data collection for the input-output of the system was accomplished. For verification of the results obtained, impact test was conducted and compared thoroughly. During input-output data was collected experimentally, sensors and actuators has been bonded and fully arranged at the link structure. This added sensors and actuators may add the dynamic load of the system which will change the resonance mode of the structure. Prior to that, the frequency response was compared thoroughly with the impact test result that has been tested on the bare link structure. Table 2 shows the results from the experimental test recorded compared with impact test result by taking the impact test result as a true value. It is noted that reasonable accuracy was obtained for experimental test result for the first three modes of vibration. Results obtained were very close to each other with percentage of error below 5 percent for all the modes of vibration. This indicates that the collected experimental input-output data were suitable to be used for further analysis of system identification and active control.

Table 2: Percentage of error for modes of vibration

\begin{tabular}{|l|l|l|l|}
\hline Resonance Mode & Impact test $(\mathbf{H z})$ & $\begin{array}{l}\text { Experiment } \\
(\mathbf{H z})\end{array}$ & $\begin{array}{l}\text { Percentage } \\
\text { of error (\%) }\end{array}$ \\
\hline 1 & 3.05 & 3.01 & 1.31 \\
\hline 2 & 18.31 & 17.64 & 3.66 \\
\hline 3 & 53.10 & 50.50 & 4.90 \\
\hline
\end{tabular}

\section{Conclusion}

This study presents experimental results concerning the single-link flexible manipulator. A new experimental test rig has been designed, fabricated and integrated. The experimental equipment's, setup and method of capturing data are elaborated. The dynamic response of the system has been obtained, input-output data was acquired and result validated. The comparative study shows a good agreement between the experimental work and impact test especially on the first mode with $1.31 \%$ of error. This validation thus affirmed the suitability of the input output data acquired for further analysis. The major contribution of this research is to highlight the selection of instrumentation system that can fulfil the demand of physical dynamic of flexible manipulator system which will be beneficial in the decentralizing control scheme for active control analysis. The experimental setup can be used later for controller development of the flexible manipulator system.

\section{Acknowledgement}

The authors wish to thank the Ministry of Education (MOE) (Vote No 4F395) and Universiti Teknologi Malaysia (UTM) for providing the research grant and facilities. This research is supported using UTM Research University grant, Vote No. 05H71.

\section{References}

[1] Abdul Razak, N. (2007). Modeling of Single-link Flexible Manipulator with Flexible Joint. Master Thesis, Universiti Teknologi Malaysia, Skudai.

[2] Ahmad, M. A. and Mohamed, Z. (2008). Vibration Suppression Techniques in Feedback Control Loop of a Flexible Robot Manipulator. Modern Applied Science. 2(2), 59 - 71.

[3] Alam, M. S. and Tokhi, M. O. (2008). Designing feedforward command shapers with Multi-objective Genetic Optimisation for vibration control of a Single-link Flexible Manipulator. Engineering Applications of Artificial Intelligence. 21(2), 229 - 246.

[4] Choi, S. B., Cho, S. S., Shin, H. C., and Kim, H. K. (1999). Quantitative feedback theory control of a single-link flexible manipulator featuring piezoelectric actuator and sensor. Smart Material and Structure. 8(1999), $338-349$.

[5] Ho, M. T. and Tu, Y. W. (2006). Position control of a Single-link Flexible Manipulator using $\mathrm{H}$-infinity based PID control. IEEE Proceedings- Control Theory and Applications. 153(5), 615 - 622.

[6] Maouche, A. R. and Attari, M. (2008). Adaptive Neural Control of a Rotating Flexible Manipulator. International Symposium on Power Electronics, Electrical Drives, Automation and Motion. 517 - 522.

[7] Mohamed, Z., Tokhi, M. O. \& Azad, A. K. M. (1996). Finite Difference and Finite Element Simulation of a Flexible Manipulator. Research Report no. 617, Department of Automatic Control \& System Engineering. University of Sheffield, UK.

[8] Phan, V. P., Goo, N. S. and Park, H. C. (2009). Vibration Suppression of a Flexible Robot Manipulator with a Lightweight Piezocomposite Actuator. International Journal of Control, Automation and Systems. 7(2), $243-251$.

[9] Physik Instrumente (PI) GmbH \& Co. (2012). P-876 DuraAct Patch Transducer. Karlsruhe, Germany.

[10]Rovner, D. M. and Franklin, G. F. (1988). Experiments in Loadadaptive Control of a Very Flexible One-link Manipulator. Automatica. 24(4), $541-548$.

[11] Rudrusamy, B. (2005). Optimal Input Shaping for Vibration Control of a Flexible Manipulator using Genetic Algorithm. Master Thesis. Universiti Teknologi Malaysia, Skudai.

[12]Ryu, J. H., Kwon, D. S. and Park Y. (2000). A Robust Controller Design Method for a Flexible Manipulator with a Large Time Varying Payload and Parameter Uncertainties. Journal of Intelligent and Robotic Systems. 27, 345 - 361.

[13]Tokhi, M.O., and Azad, A.K.M. (1996). Design and Development of an experimental flexible manipulator system. Robotica. 15, 283 292.

[14] Tokhi, M. O., Mohamed, Z. and Shaheed, M. H. (2001). Dynamic Characterization of a Flexible Manipulator System, Robotica. 19 , $571-580$.

[15] Vakil, M. (2008). Dynamics and Control of Flexible Manipulators. Doctor of Philosophy Thesis, University of Saskatchewan, Saskatoon, Canada. 